\title{
Unsupervised Control Paradigm for Performance Evaluation
}

\author{
Sathya Ramadass \\ Asst. Professor, Department of MCA \\ Jyoti Nivas College (Autonomous) \\ Bangalore, India.
}

\author{
Annamma Abraham, PhD \\ Professor and Head, Department of Mathematics \\ B.M.S. Institute of Technology \\ Bangalore, India.
}

\begin{abstract}
Intelligent control denotes the capacity to acquire and apply knowledge in control process. The important characteristics of intelligent control systems are information abstraction and knowledge-based decision making. There are different control paradigms available in the literature including Artificial Neural Networks, Fuzzy Logic Systems, Genetic Algorithms, Hybrid Models and others. This paper attempts to design open loop controller using Self Organizing Map and studies its nature and accuracy with an example.
\end{abstract}

\section{Keywords}

Competitive Learning, Intelligent Control, Rule extraction, SOM, Unsupervised Learning

\section{INTRODUCTION}

Intelligent machine rely on intelligent control (IC) in their operations. IC denotes a control technique that can be carried out using the intelligence of a human who is knowledgeable in the particular domain of control. The field of IC has grown in recent years. This is due to the capabilities of algorithm and techniques available in the area of Artificial Intelligence. IC are useful not only in autonomous complex tasks and process that needs high accuracy but, in cases of problems consist of incomplete or unexpected or unfamiliar conditions. Thus, information abstraction and knowledge-based decision making are considered important characteristics of IC. Techniques of IC include Artificial Neural Networks (ANN), Fuzzy Logic Systems, Genetic Algorithms and Hybrid Models and others [1].

Fuzzy Logic Controllers are designed based on the knowledge gathered by the experts. The process of control decisions are generated using fuzzification and defuzzification actions. A set of compositional rules are framed for control actions [2]. The other neural network controllers are built using algorithms that allow the system to learn automatically from the training patterns. There are numerous control process developed using neural networks. They are mainly classified as supervised control models, inverse controllers and adaptive controllers. The main action of controllers are to control a system or a plant when there are highly nonlinear behavior, time varying parameters and some operations are characterized in an unpredictable environment. In other words, a controller is designed to measure a variable, process it, if not suitable performs correction process and redo the operation. These systems can also be called as a closed-loop controller (CLC) because, the difference between the desired action and actual action is sent to the plant by the feedback operations. Some control systems do not take relationship or feedback from the plant to ensure the goal but apply a correction method when needed. These are called open-loop control (OLC) systems. In open-loop control system, a signal is sent to a plant and assumes that the desired results will be obtained. Sometimes a closed-loop controller acts as a openloop controller. Not all real time control applications needs to be developed with closed loop controllers. There are some areas where OLC can be useful, such as plants that shutdown automatically when crisis occur. Hence, introducing OLC overcomes the disadvantages faced in the CLC like, expensive and time-consuming process in the supervised controllers, induction of unwanted time delays, and obtaining unpredictable errors in the controller design of inverse plant controller and the possibility of stability-plasticity dilemma of Neuro-adaptive controller and others $[3,4]$. The time delay and stability-plasticity problems faced in the supervised neural network models can be easily solved in the unsupervised neural networks models. That is, a control system can be designed with Self Organizing Map (SOM) or Adaptive Resonance Theory (ART) rather than Multi Layer Perceptron Models (MLP) of ANN.

Thus, this paper attempts to design a OLC in an unsupervised learning environment more specifically SOM and studies its nature and accuracy in the field of education.

\section{UNSUPERVISED MODEL: SOM}

\subsection{KSOM}

It is nature of a human brain to map sensory inputs that makes sense to talk of one stimulus being close to another according to some metric characteristics of the stimulus. In those situations patterns present in the input vector form a kind of map where similar patterns display close to each other. These maps are called topographic feature maps. It is possible to train a neural network model with competitive learning algorithm to preserve these maps. The Kohonen network developed by Professor Teuvo Kohonen [5], represents these type of feature map. Self-organization is a case of self training. These types of training and learning are based on the biologically motivated unsupervised learning laws. It depends on the local information. In neural network, the mechanism by which the brain stores information is designed in a distributed storage of information over many units rather than centralized storage in any particular unit. Such storage should be based on the concept that long term information storage in the brain and is a consequence of adaptation in synaptic connections between neurons, whereas the activities of the neurons present the stored patterns. The networks with competitive learning behavior learn and organize information without being given correct answers for the input patterns. Thus, the problems encountered in the supervised neural networks removed here.

Kohonen Self Organizing Map (KSOM) consists of nodes arranged in a 1D or 2D array (see Figure 1). Given an input vector $x$, the SOM algorithm proceeds by identifying winning neuron in the output space. The feature map computed by the 
algorithm denotes a nonlinear transformation. Neurons in the field undergo normal competition based on a distance metric $d$

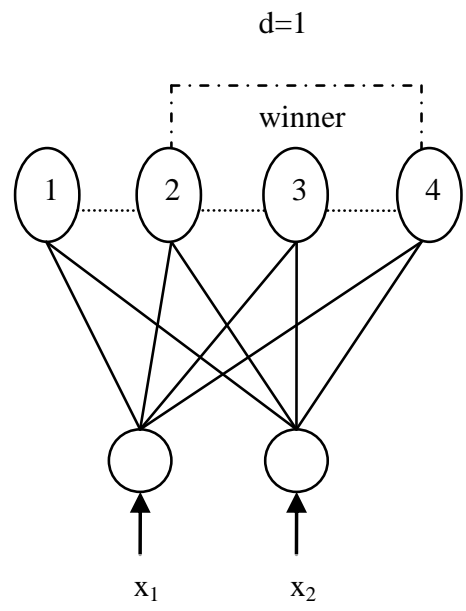

Fig 1: 1-D Kohonen SOM

The general measure to determine the winning neurons is Euclidean Distance. It employs soft computation to update weights in a planar field of neurons. In other words, a neuron that wins the competition tends to excite the neurons called cooperating neurons in its immediate neighborhood $\left(h_{j i}\right)$ more than those far away from it. The lateral distance $(d)$ between the winning neuron and the excited neuron tends to increase with the neighborhood decaying to zero. In addition, the size of topological neighborhood which is centered around winning neuron shrinks with time. Weight updates $(w)$ take place in a cluster of neurons in the neighborhood of the winner as :

$$
w_{\text {new }}=w_{\text {old }}+\eta h\left(x-w_{\text {old }}\right)
$$

The radius of the neighborhood and the learning rate $(\eta)$ reduce as iteration pass (see Figure 2). The network goes through a short ordering phase followed by convergence phase. In the convergence phase the neighborhood radius is maintained as one or zero and the learning rate parameter is maintained at a small value say 0.01 otherwise feature map will receive a topological defect. Gradual decrease of learning rate provides networks' stability.

Normally, the weight vectors of the process are initialized randomly. The problem in this method is during training some weight vectors will be so far away from any input vector that they will never be trained. Instead of random assignment, all initial weights can be set to $1 / \sqrt{ } d$ where $d$ is the number of input neurons and also the number of components in the weight vector $[6,7]$.

The capability of forming nonlinear projection of high dimensional into low dimensional data space of SOM has been used in scientific, control engineering and business application as an analysis method due its property $[8,9,10]$.

Similarly, the self adaptation nature of SOM would be a suitable technique for a OLC since OLC does not need a feedback/supervisor to ensure the correctness of the plant. One of the main characteristics of IC is extraction of knowledge from the system which can be used for controlling or decision making. In that aspect, the rule/knowledge is extracted from KSOM to perform the control process.

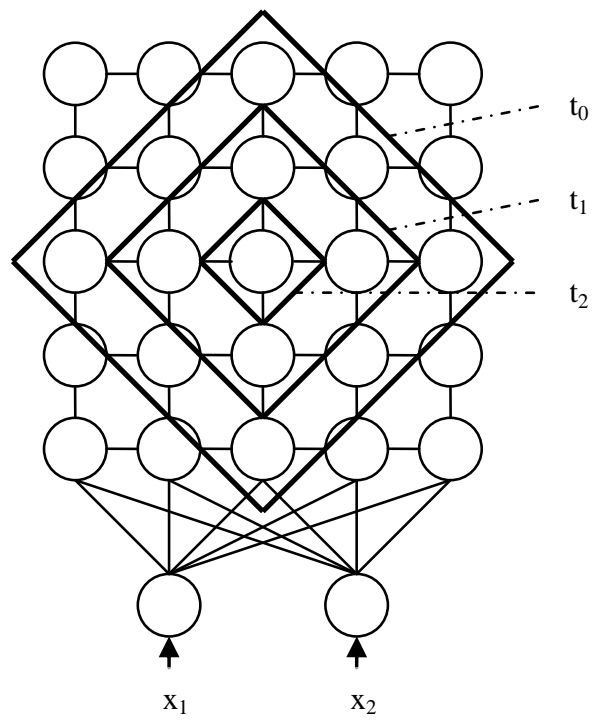

Fig 2: 2-D Kohonen SOM

\subsection{Rule extraction by Direct Method}

The knowledge used in the adaptation process of KSOM is obtained by rule extraction process. The rule extraction of SOM can be done either by boundaries approach using UMatrix (unified distance matrix) or using cluster approach. In boundary approach, U-matrix is used to find the boundary between the neighboring cluster units which leads to extraction of rules that describe the discovered pattern in the input vector [11]. In cluster approach, rule extraction is performed from a SOM by discovering clusters instead of boundaries [12]. It finds the most significant value for each feature and cluster and extracts the rule for those clusters. Thus to extract knowledge direct method approach is used from a cluster node in SOM [6]:

1. For each cluster node $j$, search for a set of attributes/patterns with each pattern $p$ satisfying the following condition: The summed weights of $p$ are the maximum among all clusters for cluster $j$, and $w_{j i} \geq w_{k i}$ for every $i \in A_{T}-A_{p}$ and for every $k \neq j$, where $A_{p}$ be the set of attributes involved in $p$ and $A_{T}$ is the set of all attributes.

2. Refine the extracted patterns or attributes in each cluster node $j$, if $A_{p}$ participates in more than one cluster.

As a first step, a set of training data is presented to the SOM system at the input layer and the connection weights are adjusted through competition among the nodes in the output layer as mentioned above. Also, neighborhood neurons also adjusted according to the winning neuron at the output layer. After some epochs, the system reaches the convergence state and now ready for the real data. The important point to be considered is the system organizes itself and learns on its own and preserves its structure. The patterns are now clustered in the competitive neurons in the output layer. After generalization, the cluster node undergoes the rule extraction procedure described above.

The algorithm finds the attributes of the input set that are significant to characterize each cluster or class. It calculates for each cluster node the activation of each attribute summed weight and highlight the highest value for each row ( Table 1). 
In order to find the attributes that are most influencing for a description of a cluster, these significant values of the attributes are normalized in percentage of the total sum of significant values of a class [13]. Then these are arranged in decreasing order. From this, the attributes with the largest significant value in the ordered sequence are taken until the cumulative percentage equals or exceeds a given threshold value. The standard threshold value is $50 \%$. Thus, these attributes and any attribute, which are highlighted as highest significant value and not considered so far, are high influence attributes on that class (see Table 2). In other words these could be the knowledge that is necessary for a controller to control the plant operation [14].

Table 1. Summed weights of each Competitive Unit

\begin{tabular}{|c|c|c|c|}
\hline $\mathbf{S M}$ & $\mathbf{C U}_{\mathbf{1}}$ & $\mathbf{C U}_{\mathbf{2}}$ & $\mathbf{C U}_{\mathbf{3}}$ \\
\hline $\mathrm{A}_{1}$ & $\mathbf{4 . 9}$ & 3.8 & 2.6 \\
\hline $\mathrm{A}_{2}$ & $\mathbf{5 . 2}$ & 2.9 & 2.7 \\
\hline $\mathrm{A}_{3}$ & $\mathbf{6 . 7}$ & 5.8 & 3.5 \\
\hline $\mathrm{A}_{4}$ & $\mathbf{5 . 9}$ & 1.5 & 3.1 \\
\hline $\mathrm{A}_{5}$ & $\mathbf{8 . 4}$ & 5.8 & 2.4 \\
\hline $\mathrm{A}_{6}$ & 0.0 & $\mathbf{3 . 0}$ & 0.2 \\
\hline $\mathrm{A}_{7}$ & 0.8 & 1.3 & $\mathbf{3 . 3}$ \\
\hline $\mathrm{A}_{8}$ & 1.4 & $\mathbf{2 . 1}$ & 1.1 \\
\hline $\mathrm{A}_{9}$ & 0.2 & $\mathbf{0 . 6}$ & 0.2 \\
\hline $\mathrm{A}_{10}$ & 3.3 & $\mathbf{4 . 2}$ & 2.8 \\
\hline
\end{tabular}

The algorithm is performed on all clusters and provides for each cluster the set of significant attributes to be used in a meaningful description of that class. Thus, number of rules describes a class and attributes are selected by the above procedure. These rules may be too strong or too soft to describe a class. This can be generalized by finding the significant attribute $A_{p}$ in the range [ min, max]. Sometimes, a rule can belong to two classes (step 2). In that case, a finer description about the class boundary between the two classes is necessary or the summed weights of $p$ in conjunction with any pattern based on the attributes can be included in the rule.

Thus, from this direct method of rule extraction, the knowledge that is used in the cluster formation of competitive units is extracted. This can be formed as a if then rule that can be used as a control mechanism of the system that need to be controlled. So, SOM algorithm along with rule extraction process can be a best technique for a open loop controller.

To find the practical efficiency and accuracy of this unsupervised controller, we tested this approach in the admission process of higher education course in a college.

\section{IMPLEMENTATION}

Education plays a vital role in human resource development of a country by creating skilled manpower, enhancing industrial productivity and improving the quality of life.

Hence, education is considered as a key factor in assessing a country's prosperity. The past few decades have seen a number of changes and reformation in the field of education due to an increased awareness and availability of information in the society $[15,16,17]$.

Table 2. Significant attributes for Competitive Unit 2

\begin{tabular}{|c|c|c|}
\hline \multicolumn{3}{|c|}{ Competitive Unit 2 } \\
\hline Features & $\begin{array}{c}\text { Significance } \\
\text { \% }\end{array}$ & $\begin{array}{c}\text { Cumulative } \\
\text { \% }\end{array}$ \\
\hline $\mathrm{A}_{3}$ & 18.65 & 18.65 \\
\hline $\mathrm{A}_{5}$ & 18.60 & 37.25 \\
\hline $\mathbf{A}_{\mathbf{1 0}}$ & 13.64 & 50.89 \\
\hline $\mathrm{A}_{1}$ & 12.14 & 63.03 \\
\hline $\mathbf{A}_{\mathbf{6}}$ & 9.69 & 72.72 \\
\hline $\mathrm{A}_{2}$ & 9.32 & 82.04 \\
\hline $\mathbf{A}_{\mathbf{8}}$ & 6.91 & 88.95 \\
\hline $\mathrm{A}_{4}$ & 4.96 & 93.91 \\
\hline $\mathrm{A}_{7}$ & 4.26 & 98.17 \\
\hline $\mathbf{A}_{\mathbf{9}}$ & 1.83 & 100 \\
\hline
\end{tabular}

Now-a-days people assess the value of education in the same manner as they assess the value of land or of share in the stock market. Parents and students want to get only such education as it would enable the student to earn more or it is seen as an asset. Students enroll for post graduation either to pursue a subject they enjoy or to get distinct advantages over other job applicants or to improve carrier prospects to hit the recession. Students are admitted for post graduation under two categories: Management and General quota. Thus, each institution will have an even spread of students of all categories.

Once the student is admitted in to an educational institution, it is the responsibility of that institution to impart the type of education that enables them to acquire knowledge and compete with students of other institutions. Thus both the educational institutions as well as the prospective students are free to assess each other mutually based on certain standard criteria as mentioned above.

In the present scenario, introducing an intelligent control system will improve the quality of education as well as performance of students in Post-Graduate (PG) levels. Keeping these aspects in focus, this paper considered two of the most valued courses in PG stream in India viz., MBA and MCA to test the KSOM as a controller.

A two layer KSOM is designed with 10 input vectors representing admission criteria and three output competitive units that represent the performance of the student during the admission about their passing grade [9]. Once the students of similar pattern are clustered into the output units, the rule extraction algorithm is applied to extract the knowledge gained in the output units, which can act, as control rules (see Table 1 and Table 2). Thus, the rules that are extracted are available to control process to evaluate the student's performance (see Figure 3) [14]. 


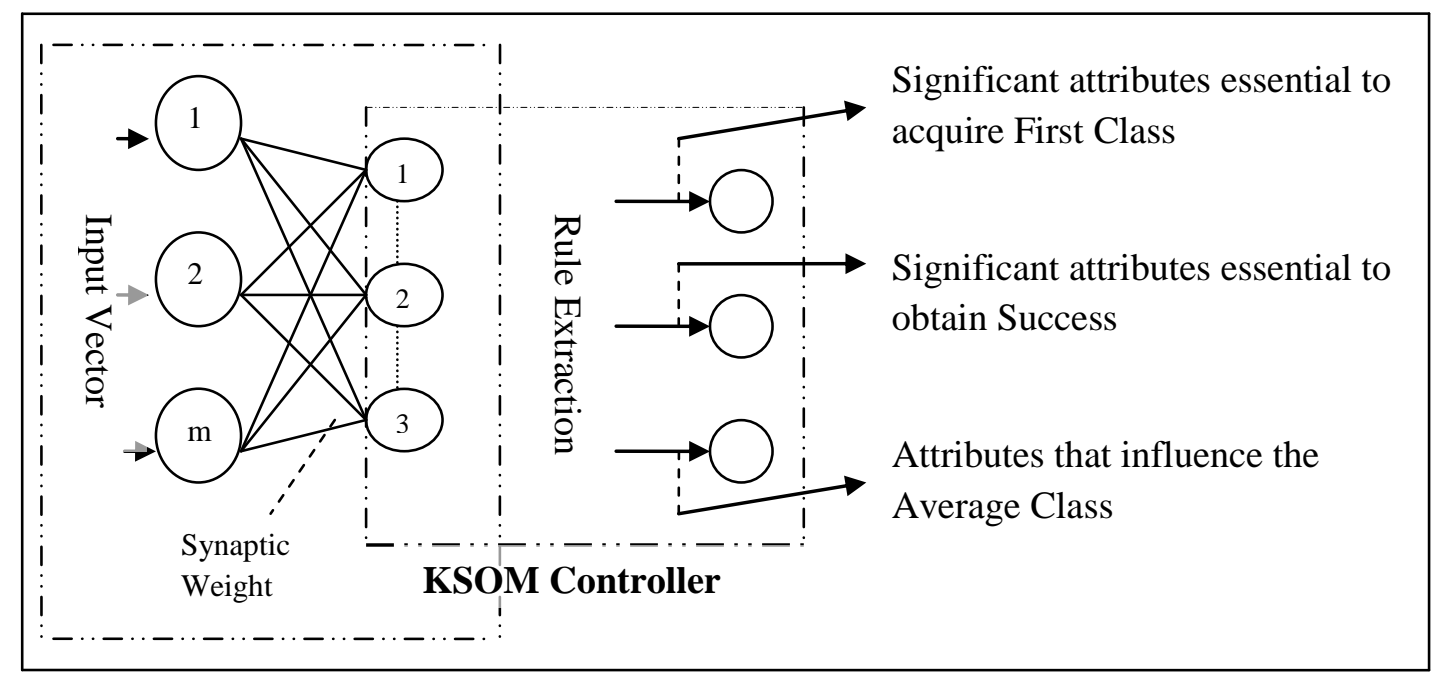

Fig 3: Architecture of KSOM Controller

\section{RESULTS AND DISCUSSION}

The evaluation scheme provides the details of the students' ability, and so, the management becomes aware of their students attitude and is proactively equipped to guide them in a proper path. Thus, the control system makes the institute/college aware that
* Similar nature of the students are to be grouped together
* Students have good academic score achieve high success rate
* Students from city perform better compared to others
* Under-privileged students needs extra attention
- Students who does not possess commercel mathematics major in the graduation/higher secondary but having work experience also performs well

Thus the intelligent control experimented in this work describes the performance of a student and recognize their potentials in a simple environment that has minimum attributes to be controlled. The systems accuracy is tested with known available data sets and found it is high and can be further improved by adjusting the input parameters.

\section{CONCLUSION}

Experiments based on the data sets showed that unsupervised learning algorithm is able to produce interpretable rules with high prediction capabilities. The scope of this research is characterized by the students' skills and is restricted to test the suggested methodologies in a college for professional courses such as MBA and MCA. The use of attribute selection method enables us to used KSOM as a controller and thus enables to improve performance of the students as well as the rank of the educational institutions. The effectiveness of the method has been analyzed with our empirical experimental results. Based on this study, it is proved that the KSOM controller have higher accuracy and feasibility. Thus, this model can be used for any number of samples and the outcome will not involve errors due to human intervention.

\section{ACKNOWLEDGMENTS}

I would like to thank Mother Teresa Women's University, Kodaikanal and the Director of PG Courses of Jyoti Nivas College (Autonomous), Bangalore for their support and continuous encouragement given to me in pursuing this research.

\section{REFERENCES}

[1] Karray, F.O., and Silva, C.D. 2004. Soft Computing and Intelligent Systems Design Theory, Tools and Applications. Pearson Education.

[2] Passino, K.M. 2001. Intelligent Control: An Overview of Techniques, Chapter in: T. Samad, Ed., "Perspectives in Control: New Concepts and Applications", IEEE Press, NJ.

[3] Antsaklis, P.J. 1999. Intelligent Control, Encyclopedia of Electrical and Electronics Engineering, 10, 493-503, John Wiley \& Sons, Inc.

[4] Sathya, R, and Manohar, G.T. 2008. Introducing Intelligent Control Paradigms to Potential Researchers. J. Jyoti Research Academy. 2 (Dec 2008), 7-12.

[5] Kohonen, T. 1998. Neurocomputing. 21, Issue 1-3.

[6] LiMin Fu. 2003. Neural Networks in Computer Intelligence. Tata McGraw-Hill Publishing Company Limited.

[7] Herbst, M., Gupta, H.V., and Casper, M.C. 2009. Mapping Model Behaviour using Self-Organizing Maps. Hydrol. Earth Syst. Sci., 13, 395-409.

[8] Aroui, T., Koubaa, Y., and Toumi, A. 2009. Clustering of the Self-Organizing Map based Approach in Induction. Machine Rotor Faults Diagnostics. Leonardo Journal of Sciences, 15, 1-14.

[9] Sathya, R., and Abhraham, A. 2010. Application of Kohonan SOM in Prediction. In the Proceeding of ICT conference. CCIS 101. Springer-Verlag Berlin Heidelberg, 313-318.

[10] Asif, U. K., Bandopadhyaya, T.K., and Sudhir, S. 2009. Classification of Stocks Using Self Organizing Map. J. Soft Computing Applications. 4, 19-24.

[11] Andrews, R., and Geva, S. 2000. Rule Extraction From Local Cluster Neural Nets. Neurocomputing.

[12] Steiner, M.T.A., Neto, P.J.S., Soma, N.Y., Shimizu, T., and Nievola, J.C. 2006. Using Neural Network Rule 
Extraction for Credit-Risk Evaluation. J. IJCSNS, 6 (5A).

[13] Kamruzzaman, S.M., and Md., M., Islam. 2006. An Algorithm to Extract Rules from Artificial Neural Networks for Medical Diagnosis Problems. Int. J. Information Technology, 12 (8).

[14] Sathya, R., and Abraham, A. 2012. Rule Extraction from SOM for Academic Evaluation, In the Proceedings of ICACII, Lecture Notes in Information Technology. 10, 184-189.

[15] Naganathan, E.R, Venkatesh, R., and Uma Maheswari. 2008. Intelligent Tutoring System: Predicting Students
Results Using Neural Networks. J. Convergence Information Technology, 3(3), 22-26.

[16] Karamouzis, S.T., and Vrettos, A. 2008. An Artificial Neural Network for Predicting Student Graduation Outcomes. In the Proceedings of the WCECS 2008.

[17] Oladokun, V.O., Adebanjo, A.T., and Charles-Owaba, O.E. 2008. Predicting Students' Academic Performance using Artificial Neural Network: A Case Study of an Engineering Course. The Pacific Journal of Science and Technology, 9, 72-79. 